\title{
Wireless Operation Monitoring and Remote Control System Implementation Using the Smartphone-Based Video Camera
}

\author{
Woongsik Kim \\ Konyang University, Konyang University, Medical IT Engineering \\ \#119 Daehang-ro, Nonsan, Chung-nam, South Korea \\ 82-41-730-5668 \\ wskim@konyang.ac.kr
}

\begin{abstract}
In this paper, we show the wireless operation monitoring and remote control system implementation using the smartphonebased video camera. The injection molding machine operation is monitored through a video camera and the defective product is determined by the pattern comparison of the injection product, so that more accurate and quantitative inspection and statistical processing can be performed for the total quality control. This study developed a product that can improve productivity and reduce cost by utilizing existing facilities. It is a product that can be developed according to production equipment and products of each company and can be customized according to characteristics of company. $\mathrm{t}$ can be used for the development of production facilities capable of real-time monitoring and control using mobile devices such as smartphones and tablet PCs, which have been actively applied recently.
\end{abstract}

\section{CCS Concepts}

- Information systems-> information system applications-> mobile information processing system.

\section{Keywords}

remote control, monitoring, smart phone, video camera, wireless control system

\section{INTRODUCTION}

The production facilities of recent age tend to be more automated or semi-automated. However, there are numerous defects in plastic auto parts in their molding process due to undesirable shapes, burr and surface defects as well as foreign objects and malfunctioning during the operation of molding, causing low productivity or low earning. Since the person in charge must remain at the production site in order to identify and detect the defects in the injection molding, studies on remote control of defect detection is needed to improve the work efficiency and productivity.

Permission to make digital or hard copies of all or part of this work for personal or classroom use is granted without fee provided that copies are not made or distributed for profit or commercial advantage and that copies bear this notice and the full citation on the first page. Copyrights for components of this work owned by others than ACM must be honored. Abstracting with credit is permitted. To copy otherwise, or republish, to post on servers or to redistribute to lists, requires prior specific permission and/or a fee. Request permissions from Permissions@acm.org.

ICMLC 2017, February 24-26, 2017, Singapore, Singapore.

(C) 2017 ACM. ISBN 978-1-4503-4817-1/17/02 ..\$15.00

DOI: http://dx.doi.org/10.1145/3055635.3056630
We have studied wireless operation condition monitoring and remote control system using smartphone-based video camera to overcome such problems.

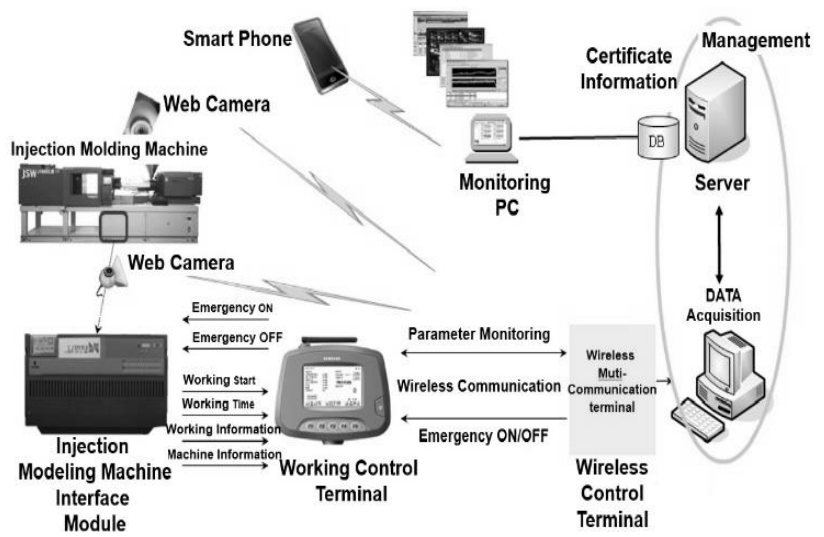

Figure 1. System configuration.

The overall system configuration of this study is shown in Fig. 1. The injection molding machine operation is monitored through a video camera and the defective product is determined by the pattern comparison of the injection product, so that more accurate and quantitative inspection and statistical processing can be performed for the total quality control.

In addition, if the operator is away, any abnormal occurrences in the injection molding machine is transmitted to the manager through the social media automatically, and the manager can immediately check the operation site using the smartphone images.

The operation status of the injection molding machine monitoring and the on / off operation are performed remotely using a smart phone, thereby improving work efficiency and reducing the incidence of defective products.

Such system can be applied to various sectors such as production, design and development in other fields.

This system consists of a monitoring terminal for real time management of injection molding machine operation, a terminal that stores and transmits image data, and a smartphone app that checks, manages and control when any malfunctioning occurs.

Chapter 2 of this study states its operation condition management system, communication protocol module for wireless communication gateway, hardware and software configuration, and the development of the smartphone app. Chapter 3 shows the actual implementation of the system. Chapter 4 concludes with the 
direction of system development and how it can interoperate with other sectors in the future.

\section{MAIN}

This chapter states the integrated system development to control and manage the system remotely. The integrated monitoring system configuration is shown in Figure 2. This system provides real-time monitoring technology as well as the notification through a smartphone in case of trouble (accident). Its integrated wired and wireless image management system provides images for determination of situation at the site so that the operator can easily monitor, manage and control to production equipment.

This system has a wireless multi-communication terminal that monitors conditions of each equipment, a server that stores, manages and supervises the received data, and a smartphone app that identifies the malfunction and makes job orders.

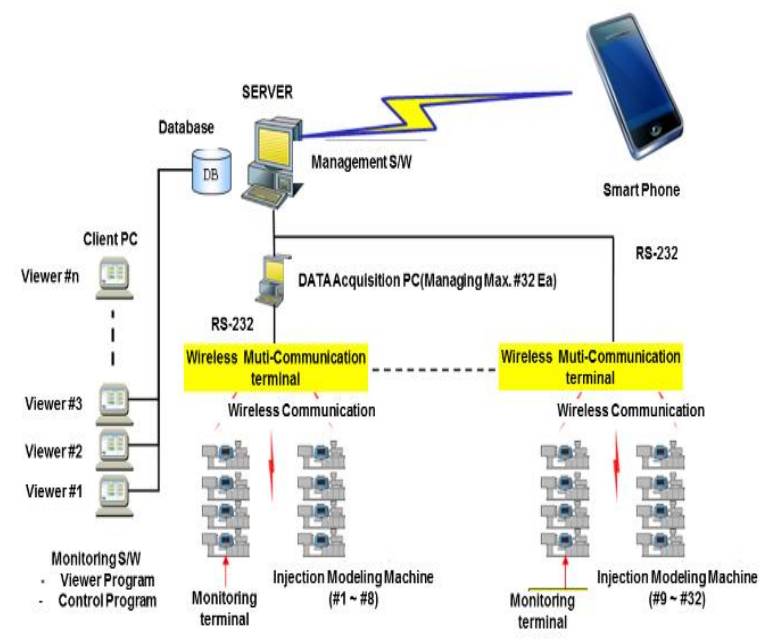

Figure 2. integrated monitoring system.

The development of this study interoperates with wit wireless operation condition monitoring/remote control system through the camera, its server, smartphone app for remote monitoring/supervision/control, wireless communication gateway module to monitor and communicate the working condition of each production equipment, and the server system that monitor, stores and control the working equipment.

Client-PC performs communication on session between the wireless multi-communication terminal, User ID, Authentication, Data transfer and Server. It also performs the data storage and processing of monitoring terminal, and communication and authorization with the Server.

Server maintains DB on general company information, operation information and equipment data and performs supervision and control through communication with the Client-PC. The steps to maintain the session is omitted if the internet address assigned to the Client-PC is fixed. However, the following steps should be considered to maintain the session if it uses the NAT(Network Address Translator). In case of a private IP, it provides information and control functions to maintain the session between the Client- PC and USER and provides the hole punching function to simply maintain the session. It currently provides TCP connection only but it can be expanded to add the UDP function in the future. It transmits the time information periodically to set the Client-PC time. Server, Client-PC, and User internet communication is shown in Figure 3.

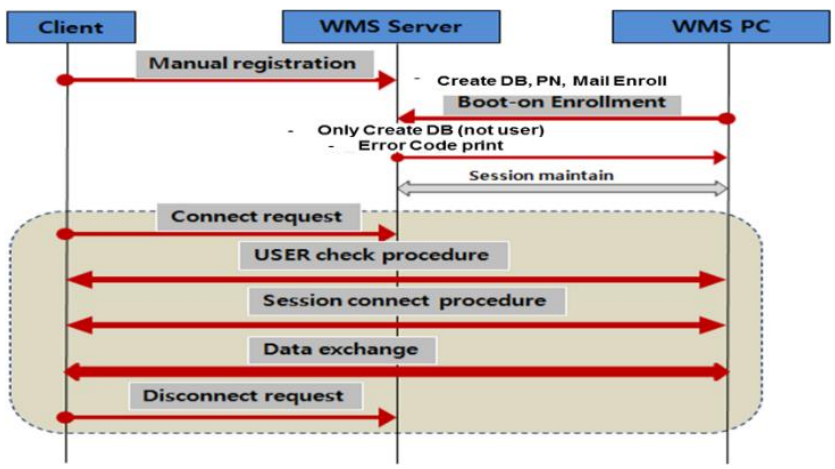

Figure 3. Server, Client-PC and User communication flow.

This system provides the Encryption Mode for the information protection. A telephone number is encrypted as a Key field depending on its Mode and the data is encrypted accordingly to the Key. Received Key is compared to the encrypted telephone number saved depending on its Mode and data is decrypted to be used accordingly to the Key. Encryption message flow is shown in Figure 4 .

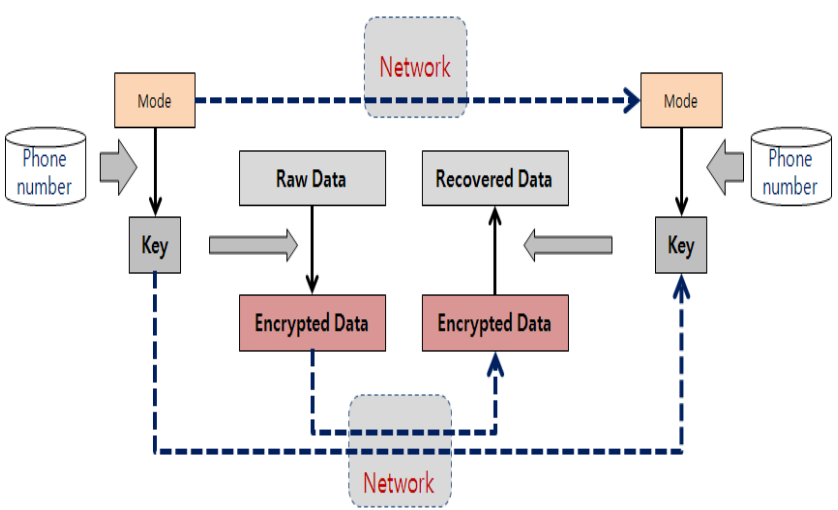

Figure 4. Encryption message flow.

Transceiver in Figure 5 communicates message with Ethernet or Console. It recognizes the received message and saves in the command saving list and transmits to the Job Distributor using the Message Queue. The result from the Distributor is received through the Message Queue and confirms the command validity period to transmits it to the server and Client-PC.

Distributor analyzes message received from the Transceiver, performs the command, identifies its function and distributes to each module. Its result is transmitted to the Transceiver. Node Controller is a functional module to communicate with the sensor node using the image data processing driver. Gateway Controller is a functional module to process commands that applies to the Gateway. VIDEO Controller is functional module to control the video functions.

Wireless communication terminal gateway consists of main board and interface board. 
Table 1. Division Description

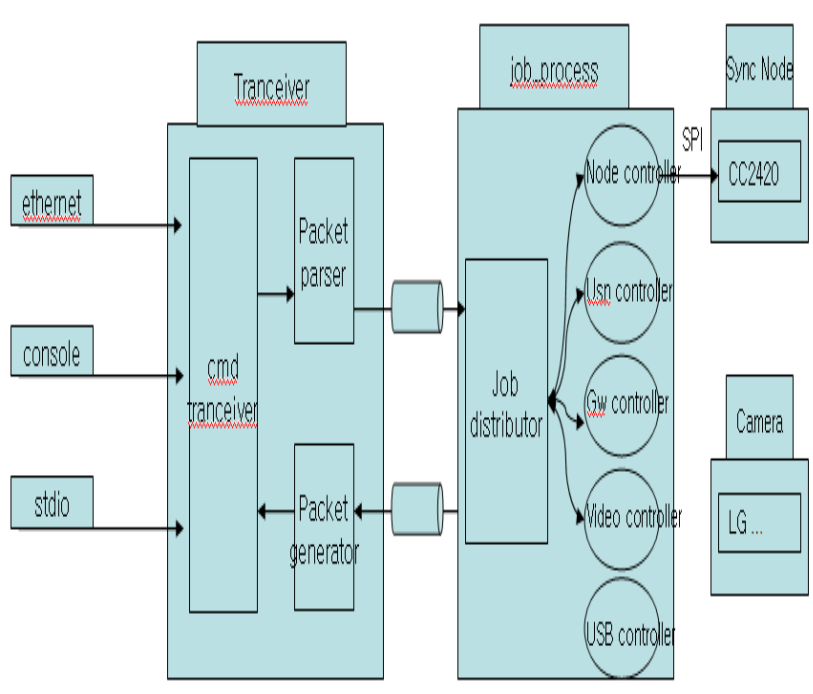

Figure 5. Gateway system Block Diagram.

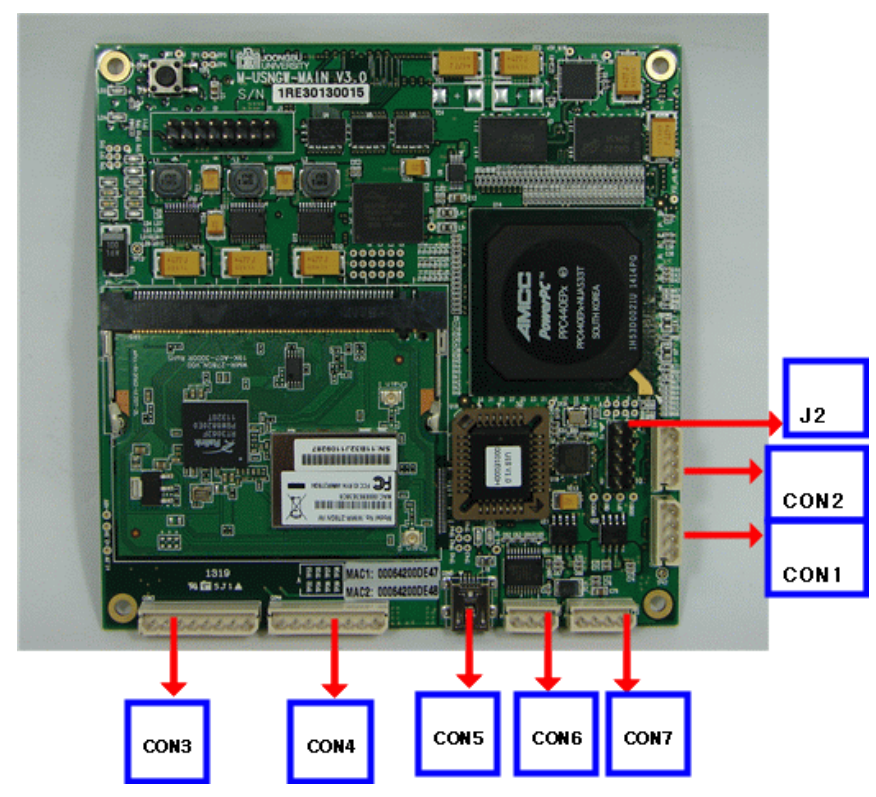

Figure 6. Wireless Communication terminal.

The contents of each pin in Figure 6 are shown in Table 1.

The initial boot state of the system is as follows. When the system is initialized, mount the flash area as a Flash file system and boot to the root file system first. When the system is initialized, the initial monitoring camera is activated. The initial boot state of the system activates the wireless LAN driver and recognizes the AP registered in the system and connects the wireless LAN. Load the driver to the monitoring camera and operate it, and transmit the camera image through channel 1 . Load all USN related drivers. It tells the GUI that the system has finished booting.

\begin{tabular}{|c|c|}
\hline Division & Description \\
\hline CON3 & $\begin{array}{c}\text { Ethernet PORT 1: ETHERNET } \\
\text { COMMUNICATION }\end{array}$ \\
\hline CON4 & $\begin{array}{c}\text { Ethernet PORT 2: ETHERNET } \\
\text { COMMUNICATION }\end{array}$ \\
\hline CON5 & USB Port1: Connect USB storage device \\
\hline CON6 & Used for debugging with Console Port \\
\hline CON7 & DC Power Input (+ 5V) \\
\hline CON2 & USB Port2: USB spare port \\
\hline CON1 & USB Port3: USB spare port \\
\hline J2 & CPLD image Fusing port \\
\hline
\end{tabular}

The Transiver of the gateway that receives the command requested from the GUI stores the command in the command save list. In this command transmission protocol, Command code and Sequence code are stored by creating a key based on the Command code and the Sequence code. In order to respond with the result of the execution of the sensor node or gateway that executed the requested command, the same command code and sequence that received the command are recorded in the protocol header and transmitted. The trunk of the gateway receiving the Ack checks the Command and Sequence of the Ack and finds a match with the requested command in the List and sends it to the GUI if the command matches. If the received message is not found in the command save list, the received response message is considered invalid. However, the event message is excluded.

At this time, if the requested command does not respond within 10 seconds, this command is deleted from the command save list. Also, an instruction arriving after 10 seconds is recognized as an invalid instruction and is deleted without being transferred to the GUI. The command for the request message is managed as a list, and when a response is received,Check the response time. In this case, if the response time exceeds 3 seconds. It is regarded as time. If a response is not received, the message will remain in the buffer, When it passes, it disappears.

In the current GUI, it is assumed that there is no response in the case of an instruction that has no response from the gateway.

The information about the periodic report among the response messages received from the sensor is information that can detect whether or not the sensor node is alive.

Therefore, Alive information is managed by storing it in the gateway buffer in accordance with the sensor ID every time it is received. After storing the periodic report information, the gateway transmits periodic report information to the GUI of the last received message when requesting from the GUI. In this case, if the time of receiving the last message of the sensor plus the time that the ensor should periodically transmit, plus the current time when comparing the message with the current time, the message is invalid And it is judged that there is a problem with the sensor sending the message.

In order to prevent duplication of sensor ID list, the installed sensor nodes should know the gateway information that they want to own in addition to the ID information. This information can be set up by the installer at the initial installation of the system by connecting to the sensor using the (RS-232) command on the console. 
Therefore, the gateway checks the information of the received sensor node, checks the gateway information of the sensor that transmitted the information, and transmits it to the upper part if it matches with the gateway ID of the sensor. If the gateway ID is different, the packet is discarded and the number of received data count and the number of received data error count are increased. Also, the Gateway manages the ID list to be managed by itself, and transmits the ID list when requesting from the GUI. However, it cannot be distinguished for sensors with the same node ID as the same gateway information. Therefore, the installer must input the correct information and configure the node information.

Gateway sensor node addition response payload is as follows. Payload passes all values for the ID List currently managed by the Gateway. The sensor node position creates a code value position. To verify that the value is reflected, the Gateway sends all Id lists. There is no ID list changing function in this ID list adding function. All data to be changed must be transmitted and received at the same time.

Table 2. Gateway sensor node addition response

\begin{tabular}{|l|l|l|l|l|l|}
\hline Filed & Len. & Type & Discription & Value & Content \\
\hline Payload & 2 & HEX & $\begin{array}{l}\text { Sensor } \\
\text { node ID }\end{array}$ & BYTE & $\begin{array}{l}\text { sensor node ID } \\
: 0 x 01 \sim 0 x 64\end{array}$ \\
\hline & 1 & HEX & $\begin{array}{l}\text { Sensor } \\
\text { node } \\
\text { location } \\
\text { code }\end{array}$ & BYTE & $\begin{array}{l}\text { Sensor node } \\
\text { location } \\
\text { representation }\end{array}$ \\
\hline
\end{tabular}

Transmits the video information connected to the gateway. When a status request comes in from the GUI, the Gateway collects the information of the currently operating video and sends it to the GUI.

The event report reports to the gateway when the sensor node's sensor information is in the critical area of the alarm. The gateway receiving the event transmits it to the GUI in real time to inform the sensor node that the event has occurred.

These sensors inform the manager of abnormal signals of the machine. Currently, the alarm system is divided into a warning alarm and a danger alarm. If the alarm generated by the sensor node is not automatically canceled or the confirmation message is not received from the gateway, the alarm message is continuously transmitted.

The sensor node that generated the alarm should continuously generate an alarm and report it to the gateway until it receives the alarm confirmation message from the gateway or until the alarm is canceled in the data collected by itself. At this time, the sensor node must continuously check the status of the alarm and notify the gateway without going into the sleep state and periodically reporting it.

If the GUI detects the sensor node that generated the alarm, it will take a specific action and send it to the alarm confirmation sensor node if it wants to no longer generate an alarm at the sensor node.
Table 3. Confirm image encoding

\begin{tabular}{|c|l|}
\hline Channel & \multicolumn{1}{|c|}{ Remarks } \\
\hline 1 & $\begin{array}{l}\text { The client for importing the image of channel 1 (port } \\
8554 \text { ) from the live media also uses shared memory t } \\
\text { o inform the connection status }\end{array}$ \\
\hline 2 & $\begin{array}{l}\text { The client for importing the image of channel 2 (port } \\
8555 \text { ) from the live media is used together with the sh } \\
\text { ared memory to inform the connection status. }\end{array}$ \\
\hline 3 & $\begin{array}{l}\text { The client for importing the image of channel 3 (port } \\
8556) \text { from the live media is used together with the sh } \\
\text { ared memory to inform the connection status }\end{array}$ \\
\hline 4 & $\begin{array}{l}\text { The client for importing the image of channel 4 (port } \\
8557 \text { ) from the live media is used together with the sh } \\
\text { ared memory to inform the connection status. }\end{array}$ \\
\hline
\end{tabular}

To check the video encoding status, IPC communication is performed between the "mobiapp" program and server. As the program starts, the "mobiapp" program creates shared memory using the KEY value. The key value is different for each channel. That is, when the channel is 0 , the server reads the value stored in the shared memory using the KEY value at the time of status request from the GUI.

The remote monitoring is done by the communication of the smartphone app developed in this study. Device operator can access the server where the recorded image is saved through the smartphone app and monitor the equipment status to notify the manager with an alarm in case of any emergency.

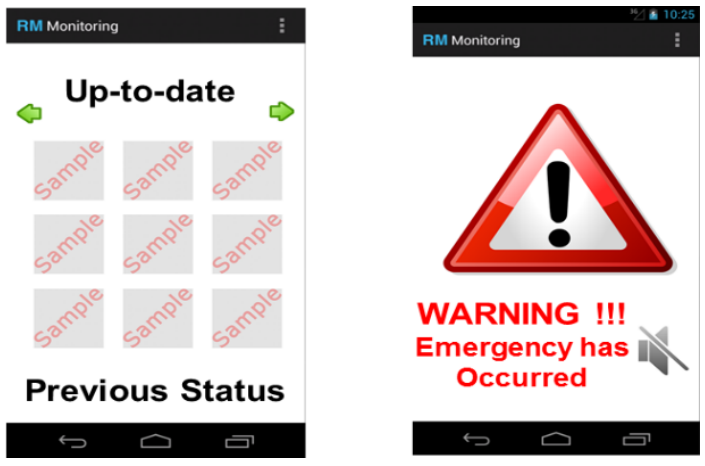

Figure 7. Smart Phone App.

The monitoring app screens in Fig. 7 show the recent and previous status. There are 9 saved images which can be enlarged after one is selected to show the equipment status. While it maintains 9 images, the oldest image is deleted if a new image is added. Above image is a test screen of sample image.

The previous status view also has 9 images in addition to the recent 9 images.

In case of an emergency at equipment, an emergency message pops open at the emergency notification screen which notifies the server and the device manager. The emergency alarm function enables a timely response to any emergency from remote location. The alarm sounds at full power and the device manager can stop the alarm with the mute button. 


\section{RESULT}

Testing on the wireless working condition monitoring and remote control system is done in this chapter using the smartphone-based camera developed in this study.

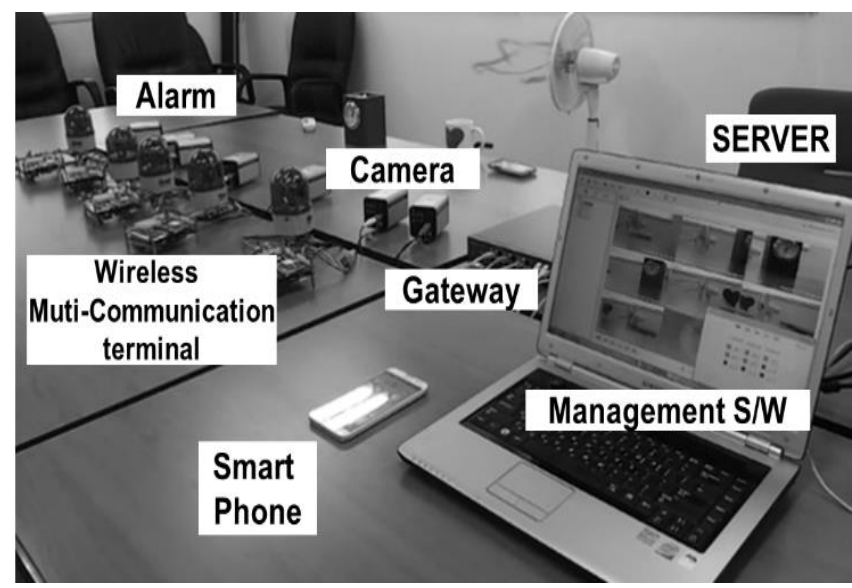

Figure 8. Wireless working condition monitoring and remote control system using the smartphone-based camera.

Figure 8 shows the system configuration: 51 mega pixel camera, Gateway, alarm indication, LED indication, manager's smartphone, managing server at remote location, and management software.

It provides with two screens per gateway so that the injection molding machine can be monitored, maintained and controled remotely from the remote operator server and manager's smartphone.

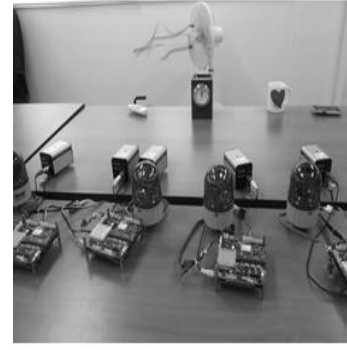

Warning

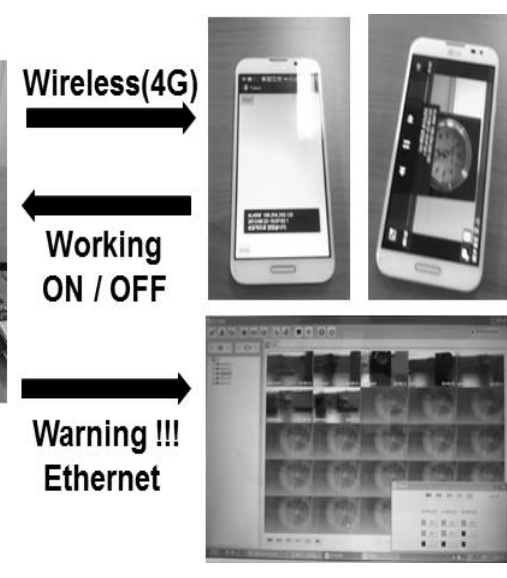

Figure 9. System operation.
In case of a problematic injection molding machine, remote operator server is notified of such failure by the gateway through the internet network and the alarm on the manager's smartphone goes off at the same time. When the operation of problematic injection molding machine is stopped using the smartphone, its $\log$ file is saved at the server. As an example of the gateway operation at the server, this system enables the LED manipulations. The server can replay and transmit the recorded video where up to 25 video can be processed. This testing shows the system is operating as intended and various control command can be applied.

\section{CONCLUSION}

This study developed a system to monitor the injection molding machine and remotely control it, and improve the performance without changing the structure of the equipment.

This system developed to monitor and control most production systems as well as injection molding machines, and it can be developed and be tailored to meet the needs of users and expand the application field. In addition, it can be developed and used as a flexible system that can be applied to other fields.

This study developed a product that can improve productivity and reduce cost by utilizing existing facilities. It is a product that can be developed according to production equipment and products of each company and can be customized according to characteristics of company.

However, if the same research is applied to the production site, it will be possible to improve the productivity by introducing the remote automation system and to develop the production facility capable of real-time response in case of trouble.

It can be used for the development of production facilities capable of real-time monitoring and control using mobile devices such as smartphones and tablet PCs, which have been actively applied recently.

\section{REFERENCES}

[1] M. Brown and D. Lowe, "Automatic Panoramic Image Stitching using Invariant Features," International Journal of Computer Vision, vol. 74, no. 1, pp. 59-73, 2007.

[2] D. Lowe, "Distinctive image features from scale-invariant keypoints." International Journal of Computer Vision, 60(2):91-110, 2004.

[3] H. Bay, T. Tuytelaars, and L.V. Gool, "SURF: Speeded Up Robust Features," 9th European Conf. Computer Vision, pages 404-417, 2006.

[4] Martin A and Robert C. Bolles, "Random Sample Consensus: A paradigm for Model fitting with Application to Image Anaysis and Automated Cartography," CACM, 24(6):381395, 1981.

[5] O. Chum and J. Matas, "Matching with PROSACProgressiveSample Consensus," Proc. IEEE Conf. Computer Vision and Pattern Recognition, pp. 220-226, 2005.

[6] Jaehyun Im and Joonki Paik, "Feature-Based Panoramic Background Generation for Object Tracking in Dynamic Video," Journal of the Institude of Electronics Engineers of Korea SP vol.45 no.6, pp 641-649, 2008.

[7] Heekyeong Jeon, Jun-mo Jeong, Kwang-yeob Lee, “An implementation of the real-time panoramic image stitching using ORB and PROSAC," 12th international SoC Design Conference, 2015.

[8] G. B. Lee, "A Study on the Economic Evaluation of Photovoltaic System," Journal of Industrial science researches, vol. 28, no. 1, pp.147-152, Aug. 2010.

[9] S. K. Firth, K. J. Lomas, and S. J. Rees, "A simple model of PV system performance and its use in fault detection," Solar Energy, vol. 84, no. 4, pp.624-635, Jun. 2010.

[10] G. H. Kim, "Designed of Intelligent Solar Tracking System using Fuzzy State-Space Partitioning Method," Journal of the Korea Institute of Information and Communication Engineering, vol. 15, no. 10, pp.2072-2078, Oct. 2011. 
[11] Agricultural Economy Research Center, "The Trend and Problems of agricultural 6th Industry in domestic and abroad ," CEO Focus No. 324.

[12] S. S. Kang, S. H. Kim, J.W Lee, H. J. Kang, "IT Convergence Technology Trend in USN based Agriculture," Electronics and Telecommunications Trens Vol. 26 No. 6, 2011. 12.

[13] National IT Industry Promotion Agency, "Guideline of construction and management for USN based agricultural crop growth and environment management system," 2010. 5.
[14] D. H. Ryu, "A Development of Urban Farm Management System based on USN," JKIECS, Vol. 8, No. 12, 1917-1922, 2013.

[15] Telecommunications Technology Association, "Greenhouse Control System- Part 4: Interface Standard between Greenhouse Operating System and Integrated Greenhouse Management System," TTAK.KO-06.0288-Part4, 2013.

[16] Ministry of Agriculture, Food and Rural Affairs, "Spreading Plan for ICT Convergence of Agricultural Food ," 2013. 8. [7] H.S Koo, J. H Min, J.Y Park, "Survey of ICT-Agriculture Convergence" Electronics and Telecommunications Trens, Vol 30. No. 2 , pp. 49-58, 2015. 